\title{
How old is too old for statins?
}

\author{
When to start, continue, or stop statins in those ages \\ $\geq 75$ years has been a quandary. Here's what 2 studies \\ have to say.
}

\section{PRACTICE CHANGER}

Do not start a statin in patients ages $\geq 75$ years who do not have known vascular disease or type 2 diabetes; start or continue a statin in all patients ages 75 to 84 with type 2 diabetes to prevent cardiovascular events and mortality; and start or continue a statin in patients ages $>75$ years who have known vascular occlusive disease.

STRENGTH OF RECOMMENDATION

B: Based on a meta-analysis of randomized controlled trials and a retrospective cohort study.

Ramos R, Comas-Cufi M, Marti-Lluch R, et al. Statins for primary prevention of cardiovascular events and mortality in old and very old adults with and without type 2 diabetes: retrospective cohort study. BMJ. 2018;362:k3359. ${ }^{1}$

Cholesterol Treatment Trialists' Collaboration. Efficacy and safety of statin therapy in older people: a meta-analysis of individual participan data from 28 randomized controlled trials. Lancet. 2019;393:407-415. ${ }^{2}$

\section{ILLUSTRATIVE CASE}

Ms. $M$ is a 76-year-old woman with wellcontrolled type 2 diabetes mellitus for 10 years and well-controlled mild hypertension. She is otherwise healthy, and her mother lived to age 95 . Ms. M has never smoked, has no previous history of vascular/cardiovascular disease, and drinks 1 glass of wine 2 to 3 times per week. Based on the American College of Cardiology (ACC) calculator, she was started on atorvastatin years ago. Is continued use of the medication of any benefit at her current age?

The 2018 American Heart Association (AHA)/ ACC/Multi-Society cholesterol guidelines do not provide primary prevention recommen- dations for those older than age 75 years. ${ }^{3}$ Up to age 75 , the guidelines recommend that patients with type 2 diabetes and a lowdensity lipoprotein cholesterol (LDL-C) level $\geq 70 \mathrm{mg} / \mathrm{dL}$, as well as those without diabetes but with an LDL-C $\geq 70 \mathrm{mg} / \mathrm{dL}$ and a 10 year atherosclerotic cardiovascular disease (ASCVD) risk $\geq 10 \%$, be started on mediumintensity statin therapy.

A 2018 consensus panel review of the current literature, sponsored by the National Institute on Aging and the National Heart, Lung, and Blood Institute, concluded that there was insufficient evidence regarding the benefits and harms of statins in older adults, especially those with comorbidities, and that there was a paucity of evidence about statin therapy outcomes (both adverse and beneficial) relevant to older adults. ${ }^{4}$

A review of all guidelines published since 2013 revealed that only the United Kingdom's 2014 National Institute for Health and Care Excellence (NICE) guideline provides a strong, risk-based recommendation for initiating primary prevention with statins in patients > 75 years old. ${ }^{5}$ These recommendations are based on the QRISK2 calculator (which has since been updated to the QRISK3), which assigns everyone ages $>75$ years a $>10 \% 10$-year risk score. This provides a universal statin indication for anyone in the 76-to-84 age range. ${ }^{6}$

Both the ACC/AHA and US Preventive Services Task Force guidelines clearly state that there are too few data and inadequate evidence in people older than 75 for a strong, risk-based statin recommendation. ${ }^{5}$ The Canadian Cardiovascular Society guideline
Bob Marshall, MD, MPH, MISM, FAAFP; Nick Bennett, DO, MBA, FAAFP

Madigan Family Medicine Residency, Tacoma, WA

DEPUTY EDITOR

Corey Lyon, DO

University of Colorado

Family Medicine Residency, Denver 


\section{$>$}

Statin therapy seems to provide no benefit to patients ages $>75$ years without ASCVD or in patients ages $\geq 85$ years without ASCVD, regardless of type 2 diabetes status. takes a similar stance, emphasizing that the recommended Framingham risk model is not well validated in people $>75$ years. $^{5}$

\section{STUDY SUMMARIES}

\section{Two different looks at} statin use in the elderly

I A retrospective cohort study ( $\mathrm{N}=46,864$; median follow-up, 5.6 years) examined whether statin treatment is associated with a reduction in atherosclerotic disease and mortality in old and very old adults with and without type 2 diabetes. ${ }^{1}$ Patients were enrolled from a large, anonymized national database in Spain. The researchers looked only at first-time users of statins and those without a statin prescription within the past 18 months.

Patients with previous ASCVD, type 1 diabetes, previous lipid-lowering treatment, dementia, cancer, or paralysis were excluded, as were those who were in residential care, were on dialysis, or had received an organ transplant. Patients were stratified by age (75-84 years and $\geq 85$ years), diabetes status (with or without type 2 diabetes), and statin use (nonuser or new user).

I Results. For patients with type 2 diabetes, the risk of ASCVD (a composite of coronary heart disease and stroke) was lower among those who took statins than among those who did not in the 75-to-84 group (hazard ratio $[\mathrm{HR}]=0.76 ; 95 \%$ confidence interval [CI], 0.65-0.89; 1-year number needed to treat $[\mathrm{NNT}]=164)$. Among those who took statins, there was also lower all-cause mortality (HR = 0.84; 95\% CI, 0.75-0.94; 1-year NNT = 306). In those ages $\geq 85$ years with diabetes, the statin group did not have a lower risk of ASCVD (HR $=0.82 ; 95 \% \mathrm{CI}, 0.53-1.26)$ or all-cause mortality (HR $=1.05 ; 95 \%$ CI, 0.86-1.28).

For patients ages 75 to 84 years without diabetes, there was no difference in risk between groups for ASCVD ( $\mathrm{HR}=0.94 ; 95 \% \mathrm{CI}$, $0.86-1.04)$ or all-cause mortality ( $\mathrm{HR}=0.98$; $95 \%$ CI, 0.91-1.05). In those ages $\geq 85$ years without diabetes, there was also no difference between groups for ASCVD ( $\mathrm{HR}=1 ; 95 \% \mathrm{CI}$, 0.80-1.24) or for all-cause mortality ( $\mathrm{HR}=1$; 95\% CI, 0.90-1.11).

I A 2019 meta-analysis of randomized controlled trials (RCTs) $(\mathrm{n}=134,537)$ and RCT summary data $(\mathrm{n}=12,705)$ evaluated the safety and efficacy of statin therapy in patients ages $\geq 55$ years. ${ }^{2}$ In the group of patients ages $>75$ years $(n=14,483$; median follow-up, 4.9 years), each $1 \mathrm{mmol} / \mathrm{L}$ reduction in LDL-C was associated with significant decreased risk for major vascular events (risk ratio [RR] $=0.82 ; 95 \% \mathrm{CI}, 0.70-0.95)$ and for major coronary events ( $R R=0.82 ; 95 \% \mathrm{CI}, 0.70-0.96)$.

In subgroup analysis by the presence or absence of previous vascular disease, there was a decreased risk per $1 \mathrm{mmol} / \mathrm{L}$ LDL-C reduction of major vascular events in patients with previous vascular disease $(\mathrm{RR}=0.85$; 95\% CI, 0.73-0.98); however, there was not a significant effect in patients without previous vascular disease ( $\mathrm{RR}=0.92$; 95\% CI, 0.73 1.16).

\section{WHAT'S NEW}

Statins may be unnecessary in older adults without ASCVD or T2DM

Statin therapy reduces the risk of ASCVD and mortality in patients ages 75 to 84 with type 2 diabetes and in patients $>75$ years with known vascular disease. However, statin therapy seems to provide no benefit in patients ages $>75$ years without ASCVD or in patients ages $\geq 85$ years without ASCVD, regardless of type 2 diabetes status.

\section{CAVEATS}

\section{Retrospective cohort design leaves cause and effect equivocal}

Even though the first study was large (with more than 46,000 patients) and the median follow-up was 5.6 years, it was a retrospective cohort study. While there is clearly an association between statin therapy and reduced ASCVD and all-cause mortality in patients with diabetes ages 75 to 84 years, cause and effect cannot be unequivocally stated. However, the meta-analysis, which included RCTs, confirms the benefit of statins in secondary prevention for older patients.

The cohort study did not look at adverse effects from statin therapy in this age group, but the data from the 2019 meta-analysis did not reveal any significant risk of myopathy. 


\section{CHALLENGES TO IMPLEMENTATION}

\section{Guidelines are lacking and} discontinuing meds requires discussion The lack of supporting guidelines to treat this age group with statins remains the largest barrier to implementation. Many patients may already be taking a statin, so a discussion about discontinuing medication will need to be initiated. JFP

\section{ACKNOWLEDGMENT}

The PURLs Surveillance System was supported in part by Grant Number UL1RR024999 from the National Center For Research Resources, a Clinical Translational Science Award to the University of Chicago. The content is solely the responsibility of the authors and does not necessarily represent the official views of the National Center for Research Resources or the National Institutes of Health.

Copyright ${ }^{\circledR}$ 2020. The Family Physicians Inquiries Network. All rights reserved.

\section{References}

1. Ramos R, Comas-Cufi M, Marti-Lluch R, et al. Statins for primary prevention of cardiovascular events and mortality in old and very old adults with and without type 2 diabetes: retrospective cohort study. BMJ. 2018;362 k3359.

2. Cholesterol Treatment Trialists' Collaboration. Efficacy and safety of statin therapy in older people: a meta-analysis of individual participant data from 28 randomized controlled trials. Lancet. 2019;393: 407-415.

3. Stone NJ, Grundy SM. The 2018 AHA/ACC/Multi-Society cholesterol guidelines: looking at past, present and future. Prog Cardiovasc Dis. 2019;62:375-383.

4. Singh S, Zieman S, Go AS, et al. Statins for primary prevention in older adults-moving towards evidencebased decision-making. $J$ Am Geriatr Soc. 2018;66: 2188-2196.

5. Mortensen MB, Falk E. Primary prevention with statins in the elderly. J Am Coll Cardiol. 2018;71:85-94.

6. ClinRisk. Welcome to the QRISK3-2018 risk calculator. www. qrisk.org/three/. Accessed May 27, 2020.

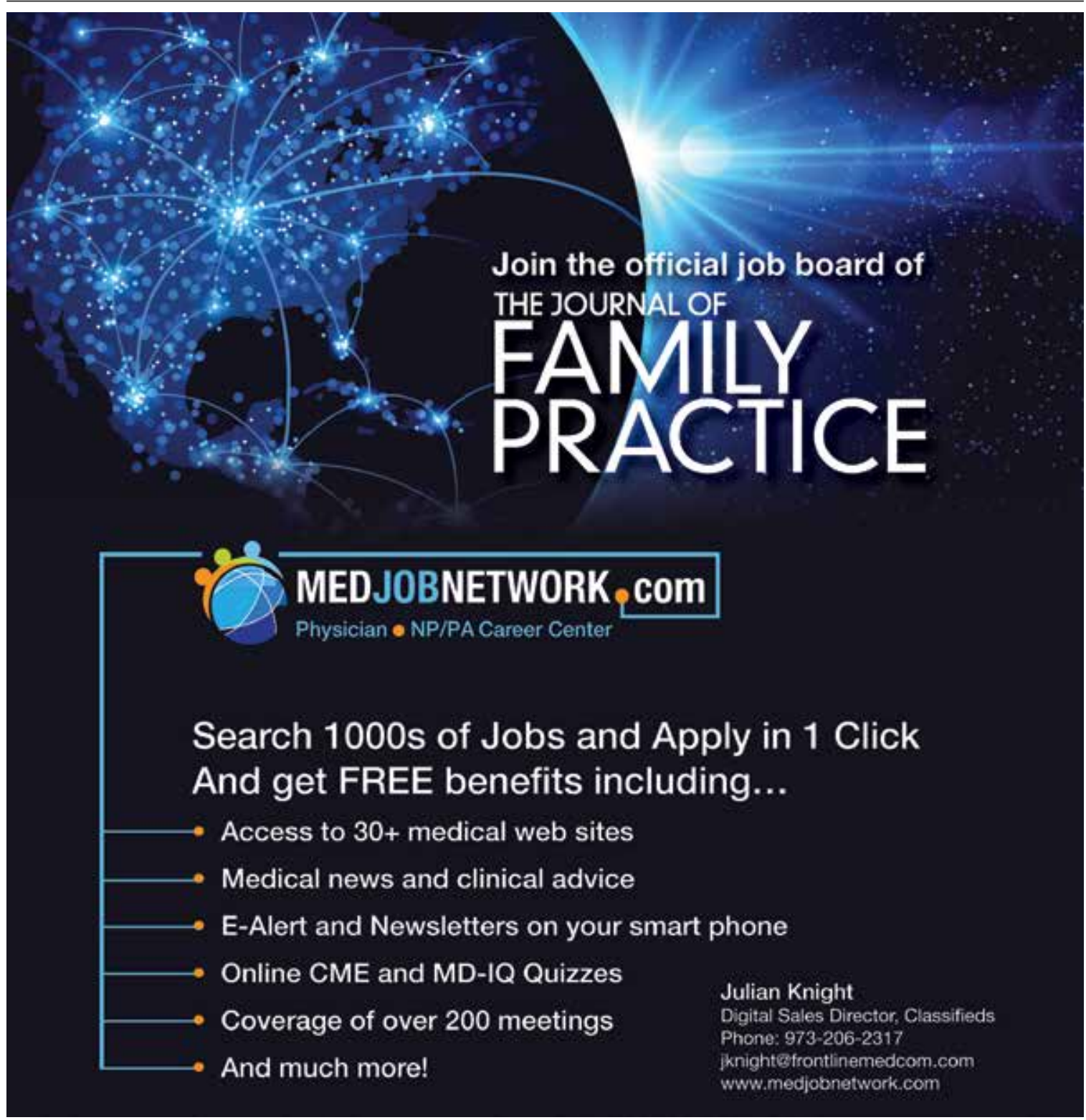

